Original Research Paper

\title{
Some Kinds of Distributional Chaos for Non-Autonomous Discrete Systems
}

\author{
${ }^{1}$ Yongxi Jiang, ${ }^{*}$ Tianxiu Lu and ${ }^{1,2}$ Xiaofang Yang \\ ${ }^{1}$ College of Mathematics and Statistics, Sichuan University of Science and Engineering, Zigong, 643000, P. R. China \\ ${ }^{2}$ Key Laboratory of Higher Education of Sichuan Province for Enterprise Information alization and Internet of Things, \\ Zigong 643000, P. R. China
}

\author{
Article history \\ Received: 10-05-2021 \\ Revised: 05-08-2021 \\ Accepted: 10-08-2021
}

Corresponding Author:

Tianxiu Lu

College of Mathematics and

Statistics, Sichuan University of

Science and Engineering,

Zigong, 643000, P.R. China

Email: lubeeltx@163.com

\begin{abstract}
In this study, several kinds of distributional chaos are defined in a non-autonomous discrete system and the chaotic behavior of the mapping sequence $f_{n, \infty}=\left(f_{n}, f_{n+1}, \cdots\right), \forall n \in \mathbb{N}$ ( $\mathbb{N}$ is a set of natural numbers) is studied. Then, the uniformly distributional chaos and maximal distributional chaos of the compound system deduced by $f_{1, \infty}$ are discussed.
\end{abstract}

Keywords: Non-autonomous Discrete Systems, Distributional Chaos, Compound System

\section{Introduction}

The definition of chaos in dynamics was started in 1975 by Li and Yorke (1975). They studied pairs of points with the property that their orbits are neither asymptotic nor separated by any positive fixed constant. To describe the complexity and unpredictability of the system from different perspectives, various definitions of chaos have been proposed, such as Devaney chaos (Banks, 1992), Generic chaos (Snoha, 1990), dense chaos (Snoha et al., 1992), dense $\delta$-chaos (Ruette, 2005), Li-Yorke sensitivity (Akin and Kolyada, 2003) and so on. While, an important extension of Li-Yorke chaos is distributional chaos, which is introduced by Schweizer and Smital (1994). The related concept distributional chaotic pair as two points for which the statistical distribution of distances between the orbits does not converge, and Schweizer and Smital (1994) proved that the existence of a single distributional chaotic pair is equivalent to the positive topological entropy (and some other notions of chaos) when restricted to the compact interval case. Since then, distributional chaos has been widely concerned in dynamical system theory (see Smítal and Štefánková, 2004; Balibrea et al., 2005; Martínez-Giménez et al., 2009; Liao et al., 2009; Oprocha, 2009; Li, 2011; Dvorakova, 2011; Wu and Chen, 2013; Shao et al., 2018). Smítal and Štefánková (2004) showed that the two notions of distributional chaos used in the paper, for continuous maps of a compact metric space, are invariants of topological conjugation. To describe distributional chaos in more detail, distributional chaos of type 1 ( $D C 1)$, of type 2 ( $D C 2$ ), and of type 3 ( $D C 3$ ) are proposed. Balibrea et al. (2004) showed that DC3 does not imply chaos in the sense of Li and Yorke. They also showed that DC3 is not invariant with respect to topological conjugation. Contrary to this, either $D C 1$ or $D C 2$ is topological conjugation invariant and implies $\mathrm{Li}$ and Yorke chaos. Then, Martinez-Gimenez (2008) provided sufficient conditions which give uniformly distributional chaos for backward shift operators, and Liao (2009) gave an example which is mixing but not distributively chaotic. In the same year, Oprocha (2009) proved that any interval map with positive topological entropy contains two invariant subsets $X, Y \subset I$ such that $\left.f\right|_{X}$ has positive topological entropy and $\left.f\right|_{Y}$ displays distributional chaos of type 1, but not conversely. Next year, Li (2011) showed that for a continuous selfmap $f$ of a compact metric space $X$ and any integer $N>0, f$ is $D C 1$ (resp. $D C 2$ ) if and only if $f^{N}$ is also $D C 1$ (resp. $D C 2$ ). And Dvorakova (2011) showed that if $f$ is a $D C 3$ continuous map of a compact metric space then also $f^{N}$ is $D C 3$ for every $N>0$. In 2013, Wu proved that the annihilation operator of an unforced quantum harmonic oscillator admits an invariant distributionally $\varepsilon$-scrambled set for any $0<\varepsilon<2$, showing that this operator can exhibit maximal distributional chaos on an uncountable invariant subset (in (Wu and Chen, 2013)). 
Moreover, the weighted shift operator exhibits uniformly distributional chaos and this property is preserved under iterations (in (Wu et al., 2013)). In 2018, Shao et al. (2018) showed that three versions named $D C 1, D C 2$ and $D C 2 \frac{1}{2}$ are invariants under iterations when $\left\{f_{n}\right\}_{n=0}^{\infty}$ is equicontinuous in $X$, which weakens the condition in the literature that $\left\{f_{n}\right\}_{n=0}^{\infty}$ uniformly converges in a compact space $X$. It is also proved that $D C 1, D C 2$, and $D C 2 \frac{1}{2}$ are invariants of topological equiconjugacy.

However, most of the literature studies chaos in autonomous systems $(X, f)$, but in reality, a lot of systems do not have good properties as autonomous systems. Different disturbances need the different functions to describe, suggesting that many systems in engineering practice are non-autonomous systems. Non-autonomous systems were first proposed by in literature (Kolyada and Snoha, 1996). Subsequently, they discussed the minimality of non-autonomous systems and the topological entropy of nonautonomous piecewise monotone dynamical systems on intervals in literature (Kolyada et al., 1999; Kolyada and Trofimchuk, 2004).

In this study, let $I=[0,+\infty]$ and the metric on $I$ is denoted as $\rho . f_{n}: I \rightarrow I, n \in \mathbb{N}$ is a mapping sequence and denoted by $f_{1, \infty}=\left(f_{1}, f_{2}, \cdots\right)$. This sequence defines a non-autonomous discrete system $\left(I, f_{1, \infty}\right)$. Under this mapping sequence, the orbit of the point $x \in I$ is

$$
\operatorname{Orb}\left(x, f_{1, \infty}\right)=\left(f_{1}^{n}(x)\right)(n \in \mathbb{N})
$$

where $f_{1}^{n}=f_{n} \circ \cdots \circ f_{1}, f_{1}^{0}$ denotes the identity mapping. Similarly, $f_{n}^{k}=f_{n+k-1} \circ \cdots \circ f_{n+1} \circ f_{n}$. If $f_{n}$ for $\forall n \in \mathbb{N}$, then $\left(I, f_{1, \infty}\right)$ is an autonomous discrete system $(I, f)$.

Next section, several definitions of distributional chaoticity are given. In section 3 and section 4, the main results are established.

\section{Preliminaries}

Similar to the definition of distributional chaos in autonomous systems, the following defines distributional chaos in the case of non-autonomous.

\section{Definition 2.1}

Let $f_{n, \infty}=\left(f_{n}, f_{n+1}, \cdots\right)(\forall n \in \mathbb{N})$ is a mapping sequence on $I$. $f_{n, \infty}$ is called distributional chaos (or distributional chaos of type 1 , briefly, $D C 1$ ), if there is an uncountable subset $S \subset I$ such that, for $\forall x, y \in S, x \neq y$,

$$
\begin{aligned}
& \text { (i) } \forall t>0, F_{x y}^{*}\left(t, f_{n, \infty}\right) \\
& =\limsup _{k \rightarrow \infty} \frac{1}{k-n+1} \sum_{i=n}^{k} \chi_{[0, t)}\left(\rho\left(f_{n}^{i-n+1}(x), f_{n}^{i-n+1}(y)\right)\right)=1, \\
& \text { (ii) } \exists t>0, F_{x y}\left(t, f_{n, \infty}\right) \\
& =\liminf _{k \rightarrow \infty} \frac{1}{k-n+1} \sum_{i=n}^{k} \chi_{[0, t)}\left(\rho\left(f_{n}^{i-n+1}(x), f_{n}^{i-n+1}(y)\right)\right)=0,
\end{aligned}
$$

where, $t \in \mathbb{R}, X_{[0, t)}$ is the characteristic function of the set $X_{[0, t)}$. That is, when $s \in[0, t), \chi_{[0, t)}(s)=1$; when $s \notin[0, t)$, $\chi_{[0, t)}(s)=0$.

$F_{x y}^{*}\left(t, f_{n, \infty}\right)$ and $F_{x y}\left(t, f_{n, \infty}\right)$ are called the upper and lower distributional functions of $f_{n, \infty}$, respectively and the uncountable subset $S$ is called the distributional scrambled set of $f_{n, \infty}$ in $I$.

The following will give other definitions of distributional chaoticity in the case of non-autonomous:

\section{Definition 2.2}

The mapping sequence $f_{n, \propto}(\forall n \in \mathbb{N})$ is called:

(1) distributional chaos of type 2 (briefly, $D C 2$ ), if there is an uncountable subset $S \subset I$, for any $x, y \in S$ and $t>0, F_{x y}^{*}\left(t, f_{n, \infty}\right)=1$ and $F_{x y}\left(t, f_{n, \infty}\right)<1 ;$

(2) distributional chaos type 3 (briefly, DC3), if there is an uncountable subset $S \subset I$, for any $x, y \in S$ and $t>0, F_{x y}^{*}\left(t, f_{n, \infty}\right)>F_{x y}\left(t, f_{n, \infty}\right)$;

(3) distributional chaos of type $2 \frac{1}{2}$, (briefly, $D C 2 \frac{1}{2}$ ), if there is an uncountable subset $S \subset I$, for any $x, y \in S$ , there exist $c>0, r>0$ such that $F_{x y}\left(t_{n}, f_{n, \infty}\right)<c<$ $F_{x y}^{*}\left(t, f_{n, \infty}\right)$ for all $0<t<r ;$

(4) $(p, q)$-distributional chaos (where $0 \leq p \leq q \leq 1$, briefly, $(p, q)$-DC), if there is an uncountable subset $S \subset I$ and $\varepsilon>0$, for any $x, y \in S, F_{x y}^{*}\left(t, f_{n, \infty}\right)=q$, $F_{x y}\left(t, f_{n, \propto}\right)=p$ for all $0<t<\varepsilon$;

(5) uniformly distributional chaos, if there is an uncountable subset $S \subset I$ satisfying that,

i. $F_{x y}^{*}\left(t, f_{n, \infty}\right)=1$ for any $x, y \in S$ and any $t>0$,

ii. there exists $t_{0}>0$ such that $F_{x y}\left(t_{0}, f_{n, \propto}\right)=0$ for any $x, y \in S$;

(6) maximal distributional chaos, if there is an uncountable subset $S \subset I$ satisfying that,

i. $F_{x y}^{*}\left(t, f_{n, \infty}\right)=1$ for any $x, y \in S$ and any $t>0$, ii. $F_{x y}\left(t_{0}, f_{n, \infty}\right)=0$ for any $x, y \in S$ and any $0<t_{0}<$ diamI . 


\section{The Relationship of Chaotic Properties between $f_{1, \infty}$ and $f_{n, \infty}$}

\section{Lemma 3.1}

$\mathrm{Wu}$ et al. (2013) the mapping sequence $f_{1, \infty}$ is distributionally chaotic if and only if the mapping sequence $f_{n, \infty}(\forall n \in \mathbb{N}, n \geq 2)$ is distributionally chaotic.

Similar to Lemma 3.1, the following discuss distributional chaos in Definition 2.2.

\section{Theorem 3.2}

The mapping sequence $f_{1, \infty}$ is $\mathcal{P}$ - chaotic if and only if the mapping sequence $f_{n, \infty}(\forall n \in \mathbb{N}, n \geq 2)$ is $\mathcal{P}$ chaotic. Where $\mathcal{P}$ - chaos stands for $D C 2, D C 3$, $D C 2 \frac{1}{2},(p, q)$-distributional chaos, uniformly distributional chaos, or maximal distributional chaos.

\section{Proof}

Only the sufficiency of the case $n=2$ is proved. The necessity is similar,

(1) For $D C 2$, let $S \subset I$ be a distributional scrambled set of $f_{2, \infty}$, then $\forall x, y \in S, x \neq y, \forall t>0$, one has

$$
\begin{aligned}
& \text { (i) } F_{x y}^{*}\left(t, f_{2, \infty}\right) \\
& =\limsup _{k \rightarrow \infty} \frac{1}{k-1}\left|\left\{2 \leq i \leq k: \rho\left(f_{2}^{i-1}(x), f_{2}^{i-1}(y)\right)<t\right\}\right|=1 \text {, } \\
& \text { (ii) } F_{x y}\left(t, f_{2, \infty}\right) \\
& =\liminf _{k \rightarrow \infty} \frac{1}{k-1}\left|\left\{2 \leq i \leq k: \rho\left(f_{2}^{i-1}(x), f_{2}^{i-1}(y)\right)<t_{0}\right\}\right| \\
& <F_{x y}^{*}\left(t, f_{2, \infty}\right) \text {, }
\end{aligned}
$$

where, $|A|$ represents the cardinality of the set $A$.

Since $f_{1}$ is surjective, then for any $x, y \in S, x \neq y$, there exist $x^{*}, y^{*} \in I, x^{*} \neq y^{*}$ such that $f_{1}\left(x^{*}\right)=x, f_{1}\left(y^{*}\right)=y$. Taking an inverse image of each element in $S$ under $f_{1}$, and let $T$ is the set of these inverse images, then $T$ is an uncountable set. The following will prove that $T$ is the distributional scrambled set of $f_{1, \infty}$.

(a) For any $t>0$ and any $x^{*}, y^{*} \in T$, let $c=1$ if $d\left(f_{1}\left(x^{*}\right), f_{1}\left(y^{*}\right)\right)<t$; let $c=0$ if $d\left(f_{1}\left(x^{*}\right), f_{1}\left(y^{*}\right)\right) \geq t$, then,

$$
\begin{aligned}
F_{x^{*} y^{*}}^{*}\left(t, f_{1, \infty}\right) & =\limsup _{k \rightarrow \infty} \frac{1}{k}\left|\left\{1 \leq i \leq k: \rho\left(f_{1}^{i}\left(x^{*}\right), f_{1}^{i}\left(y^{*}\right)\right)<t\right\}\right| \\
& =\limsup _{k \rightarrow \infty} \frac{1}{k}\left(\left|\left\{2 \leq i \leq k: \rho\left(f_{1}^{i}\left(x^{*}\right), f_{1}^{i}\left(y^{*}\right)\right)<t\right\}\right|+c\right) \\
& =\limsup _{k \rightarrow \infty} \frac{1}{k}\left|\left\{2 \leq i \leq k: \rho\left(f_{2}^{i-1}(x), f_{2}^{i-1}(y)\right)<t\right\}\right| \\
& =\limsup _{k \rightarrow \infty} \frac{1}{k-1}\left|\left\{2 \leq i \leq k: \rho\left(f_{2}^{i-1}(x), f_{2}^{i-1}(y)\right)<t\right\}\right| \\
& =F_{x y}^{*}\left(t, f_{2, \infty}\right)=1 .
\end{aligned}
$$

(b) For any $t>0$,

$$
\begin{aligned}
F_{x^{*} y^{*}}\left(t, f_{1, \infty}\right) & =\liminf _{k \rightarrow \infty} \frac{1}{k}\left|\left\{1 \leq i \leq k: \rho\left(f_{1}^{i}\left(x^{*}\right), f_{1}^{i}\left(y^{*}\right)\right)<t\right\}\right| \\
& =\liminf _{k \rightarrow \infty} \frac{1}{k}\left(\left|\left\{2 \leq i \leq k: \rho\left(f_{2}^{i-1}(x), f_{2}^{i-1}(y)\right)<t\right\}\right|+c\right) \\
& =\liminf _{k \rightarrow \infty} \frac{1}{k-1}\left|\left\{2 \leq i \leq k: \rho\left(f_{2}^{i-1}(x), f_{2}^{i-1}(y)\right)<t\right\}\right| \\
& =F_{x y}\left(t, f_{2, \infty}\right) \\
& <1 .
\end{aligned}
$$

So, $F_{x^{*} y^{*}}\left(t, f_{1, \infty}\right)<F_{x^{*} y^{*}}^{*}\left(t, f_{1, \infty}\right)$. Thus, $f_{1, \infty}$ is $D C 2$.

In the following proof, $x^{*}, y^{*}, S, T$ are the same as the proof in (1).

(2) For $D C 3$, since $F_{x y}\left(t, f_{2, \infty}\right)<F_{x^{*} y^{*}}\left(t, f_{2, \infty}\right)$ for any $t>0$ and because

$$
F_{x^{*} y^{*}}^{*}\left(t, f_{1, \infty}\right)=F_{x y}^{*}\left(t, f_{2, \infty}\right), F_{x^{*} y^{*}}\left(t, f_{1, \infty}\right)=F_{x y}\left(t, f_{2, \infty}\right),
$$

then, $F_{x^{*} y^{*}}\left(t, f_{1, \infty}\right)<F_{x^{*} y^{*}}^{*}\left(t, f_{1, \infty}\right)$.

So, $f_{1, \infty}$ is $D C 3$.

(3) For $D C 2 \frac{1}{2}$, since there exist $c>0, r>0$ such that $F_{x y}\left(t, f_{2, \infty}\right)<c<F_{x y}^{*}\left(t, f_{2, \infty}\right)$ for any $x, y \in S$ and any $0<t<r$ , then for any $x^{*}, y^{*} \in T$, for the above $c>0, r>0$, one has that

$$
F_{x^{*} y^{*}}\left(t, f_{1, \infty}\right)=F_{x y}\left(t, f_{2, \infty}\right)<c<F_{x y}^{*}\left(t, f_{2, \infty}\right)=F_{x^{*} y^{*}}^{*}\left(t, f_{1, \infty}\right)
$$

for any $0<t<r$.

That is to say, $f_{1, \infty}$ is $D C 2 \frac{1}{2}$.

(4) For $(p, q)$-distributional chaos (where $0 \leq p \leq q$ $\leq 1)$, since there exists a $\varepsilon>0$ such that

$$
F_{x y}^{*}\left(t, f_{2, \infty}\right)=q, F_{x y}\left(t, f_{2, \infty}\right)=p
$$

for any $x, y \in S$ and any $0<t<\varepsilon$, then for any $x^{*}, y^{*} \in T$,

$$
F_{x^{*} y^{*}}^{*}\left(t, f_{1, \infty}\right)=F_{x y}^{*}\left(t, f_{2, \infty}\right)=q, F_{x^{*} y^{*}}\left(t, f_{1, \infty}\right)=F_{x y}\left(t, f_{2, \infty}\right)=p,
$$

for any $0<t<\varepsilon$.

So, $f_{1, \infty}$ is $(p, q)$-distributionally chaotic.

(5) For uniformly distributional chaos, by (a), $F_{x^{*} y^{*}}^{*}\left(t, f_{1, \infty}\right)=1$ for any $t>0$. The proof of the lower distributional function is as follows. 
Since there exists a $t_{0}>0$ such that $F_{x y}\left(t_{0}, f_{2, \infty}\right)=0$ for any $x, y \in S$, then for any $x^{*}, y^{*} \in T$ and the above $t_{0}$ , one has that

$$
F_{x * y *}\left(t_{0}, f_{1, \infty}\right)=F_{x y}\left(t_{0}, f_{2, \infty}\right)=0
$$

This means that $f_{1, \propto}$ is uniformly distributional chaos.

(6) For maximal distributional chaos, by (a), $F_{x^{*} y^{*}}^{*}\left(t, f_{1, \infty}\right)=1$ for any $t>0$. The proof of the lower distributional function is as follows.

Since $F_{x y}\left(t_{0}, f_{2, \infty}\right)=0$ for any $x, y \in S$ and any $0<t_{0}<$ diamI, then for any $x^{*}, y^{*} \in T$ and any $0<t_{0}<$ diamI, one has that

$$
F_{x * y *}\left(t_{0}, f_{1, \infty}\right)=F_{x y}\left(t_{0}, f_{2, \infty}\right)=0
$$

Thus, $f_{1, \infty}$ is maximal distributional chaos.

The proof is completed.

\section{Distributional Chaoticity of Compound System of $f_{1, \infty}$}

This section mainly discusses the relationship between the distributional chaos of $f_{1, \propto}$ and the distributional chaos of the compound system $f_{1, \infty}^{[m]}$ ( $m$ is a positive integer). In the following, it is always assumed that $f_{n}(n \in \mathbb{N})$ are surjective, which is a common condition when dealing with these systems.

For any $m \in \mathbb{N}$, denote:

$$
\begin{aligned}
& h_{1}=f_{m} \circ \cdots \circ f_{1}, \\
& h_{2}=f_{2 m} \circ \cdots \circ f_{m+1}, \\
& \cdots \\
& h_{p}=f_{p m} \circ \cdots \circ f_{(p-1) m+1}, \cdots
\end{aligned}
$$

$\left(I, h_{1, \propto}\right)$ is called a compound system of $\left(I, f_{1, \propto}\right)$. To make it easier to see the relationship between $\operatorname{system}\left(I, h_{1, \infty}\right)$ and system $\left(I, f_{1, \infty}\right)$, the compound system $h_{1, \infty}$ is also denoted by $f_{1, \infty}^{[m]}$.

\section{Theorem 4.1}

If the mapping sequence $f_{1, \infty}$ is uniformly distributionally chaotic, then for any $m \in \mathbb{N}, f_{1, \infty}^{[m]}$ is also uniformly distributionally chaotic.

Proof

Let $S_{0}$ is a uniformly distributionally scrambled set of $f_{1, \infty}$. For any $m \in \mathbb{N}$, the following will prove that $S_{0}$ is also a uniformly distributionally scrambled set of $f_{1, \infty}^{[m]}$. (i) For any $x, y \in S_{0}: x \neq y$ and any $t>0$, it is claimed that there is an $i \in \mathbb{N}$ such that

$$
F_{x y}^{*}\left(t, f_{1, \infty}^{[m]}\right)=\limsup _{k \rightarrow \infty} \frac{1}{k} \sum_{i=1}^{k} \chi_{[0, t)}\left(\rho\left(f_{1}^{i m}(x), f_{1}^{i m}(y)\right)\right)=1 .
$$

In fact, hypothetically, there exist $x_{0}, y_{0} \in S_{0}: x_{0} \neq y_{0}$ and $t_{0}>0$ such that $F_{x_{0} y_{0}}^{*}\left(t_{0}, f_{1, \infty}^{[m]}\right)<1$, then,

$$
\begin{aligned}
& \limsup _{k \rightarrow \infty} \frac{1}{k m} \sum_{i=1}^{k m} \chi_{\left[0, t_{0}\right)}\left(\rho\left(f_{1}^{i}(x), f_{1}^{i}(y)\right)\right) \\
& =\limsup _{k \rightarrow \infty} \frac{1}{k m} \sum_{j=1}^{m} \sum_{i=0}^{k-1} \chi_{\left[0, t_{0}\right)}\left(\rho\left(f_{1}^{m i+j}(x), f_{1}^{m i+j}(y)\right)\right) \\
& \leq \frac{1}{m} \sum_{j=1}^{m}\left(\limsup _{x \rightarrow \infty} \frac{1}{k} \sum_{i=0}^{k-1} \chi_{\left[0, t_{0}\right)}\left(\rho\left(f_{1}^{m i+j}(x), f_{1}^{m i+j}(y)\right)\right)\right) \\
& \leq \frac{1}{m}\left(m-1+F_{x_{0} y_{0}}^{*}\left(t_{0}, f_{1, \infty}^{[m]}\right)\right) \\
& <1 .
\end{aligned}
$$

Since, $S_{0}$ is a uniformly distributionally scrambled set of $f_{1, \infty}$, then $F_{x_{0} y_{0}}^{*}\left(\frac{t_{0}}{2}, f_{1, \infty}\right)=1$. By the definition of upper limit, there is a strictly increasing sequence $\left\{n_{s}\right\}_{s \in \mathbb{N}}$ such that

$$
F_{x_{0} y_{0}}^{*}\left(\frac{t_{0}}{2}, f_{1, \infty}\right)=\lim _{8 \rightarrow \infty} \frac{1}{n_{s}} \sum_{i=1}^{n_{s}} \chi_{\left[0, \frac{t_{0}}{2}\right.}\left(\rho\left(f_{1}^{i}\left(x_{0}\right), f_{1}^{i}\left(y_{0}\right)\right)\right)=1 .
$$

Put

$$
\begin{aligned}
& M_{0}=\left\{k m: k \in \mathbb{Z}^{+}\right\} \cap\left\{n_{s}: s \in \mathbb{N}\right\}, \\
& M_{1}=\left\{k m+1: k \in \mathbb{Z}^{+}\right\} \cap\left\{n_{s}: s \in \mathbb{N}\right\}, \\
& \quad \cdots \\
& M_{m-1}=\left\{k m+m-1: k \in \mathbb{Z}^{+}\right\} \cap\left\{n_{s}: s \in \mathbb{N}\right\} .
\end{aligned}
$$

Then there exists a $l \in\{0,1, \cdots, m-1\}$ such that $M_{l}$ is an infinite set. Denote $M_{l}=\left\{n_{s}^{l}: s \in \mathbb{N}\right\}$, then

$$
\lim _{8 \rightarrow \infty} \frac{1}{n_{s}^{l}} \sum_{i=1}^{n_{s}^{l}} \chi_{\left[0, \frac{t_{0}}{2}\right.}\left(\rho\left(f_{1}^{i}\left(x_{0}\right), f_{1}^{i}\left(y_{0}\right)\right)\right)=1 .
$$

Thus,

$$
\begin{aligned}
& 1 \geq \limsup _{8 \rightarrow \infty} \frac{1}{n_{s}^{l}+m-l} \sum_{i=1}^{n_{s}^{l}+m-l} \chi_{\left[0, t_{0}\right)}\left(\rho\left(f_{1}^{i}\left(x_{0}\right), f_{1}^{i}\left(y_{0}\right)\right)\right) \\
& \geq \underset{8 \rightarrow \infty}{\limsup } \frac{1}{n_{s}^{l}+m-l} \sum_{i=1}^{n_{s}^{l}} \chi_{\left[0, t_{0}\right)}\left(\rho\left(f_{1}^{i}\left(x_{0}\right), f_{1}^{i}\left(y_{0}\right)\right)\right) \\
& \geq \underset{8 \rightarrow \infty}{\limsup } \frac{n_{s}^{l}}{n_{s}^{l}+m-l} \frac{1}{n_{s}^{l}} \sum_{i=1}^{n_{s}^{l}} \chi_{\left[0, \frac{t_{0}}{2}\right.}\left(\rho\left(f_{1}^{i}\left(x_{0}\right), f_{1}^{i}\left(y_{0}\right)\right)\right) \\
& =1 .
\end{aligned}
$$


By the definition of $M_{l}$, one can get that $m \mid n_{s}^{l}+m-l$. So

$$
\begin{aligned}
& \limsup _{k \rightarrow \infty} \frac{1}{k m} \sum_{i=1}^{k m} \chi_{\left[0, t_{0}\right)}\left(\rho\left(f_{1}^{i}\left(x_{0}\right), f_{1}^{i}\left(y_{0}\right)\right)\right) \\
& \geq \limsup _{8 \rightarrow \infty} \frac{1}{n_{s}^{l}+m-l} \sum_{i=1}^{n_{s}^{l}+m-l} \chi_{\left[0, t_{0}\right)}\left(\rho\left(f_{1}^{i}\left(x_{0}\right), f_{1}^{i}\left(y_{0}\right)\right)\right) \\
& =1 .
\end{aligned}
$$

This contradicts that

$$
\limsup _{k \rightarrow \infty} \frac{1}{k m} \sum_{i=1}^{k m} \chi_{\left[0, t_{0}\right)}\left(\rho\left(f_{1}^{i}(x), f_{1}^{i}(y)\right)\right)<1 .
$$

(ii) The following prove that there exists a $\delta>0$ such that

$$
F_{x y}\left(\delta, f_{1, \infty}^{[m]}\right)=\liminf _{k \rightarrow \infty} \frac{1}{k} \sum_{i=1}^{k} \chi_{[0, \delta)}\left(\rho\left(f_{1}^{[i m]}(x), f_{1}^{[i m]}(y)\right)\right)=0
$$

for any $x, y \in S_{0}: x \neq y$.

In fact, since $S_{0}$ is a uniformly distributionally scrambled set of $f_{1, \infty}$, there exists $\delta_{1}>0$ such that $F_{x y}\left(\delta_{1}, f_{1, \infty}\right)=0$ for any $x, y \in S_{0}: x \neq y$.

Hypothetically, there exist $x_{\delta}, y_{\delta} \in S_{0}$ such that $F_{x_{\delta} y_{\delta}}\left(\delta, f_{1, \infty}^{[m]}\right)>0$ for any $\delta>0$. In particular, for $\delta^{*}=\frac{\delta_{1}}{2}$ $>0$, there are $x_{\delta^{*}}, y_{\delta^{*}} \in S_{0}$ such that $F_{x_{\delta^{*}} y_{\delta^{*}}}\left(\delta^{*}, f_{1, \infty}^{[m]}\right)>0$.

Since $F_{x_{\delta^{*}} y_{\delta^{*}}}\left(\delta_{1}, f_{1, \infty}\right)=0$. then there exists a strictly increasing sequence $\left\{m_{s}\right\}_{s \in \mathbb{N}}$ such that

$$
F_{x_{\delta^{*}} y_{\delta^{*}}}\left(\delta_{1}, f_{1, \infty}\right)=\lim _{8 \rightarrow \infty} \frac{1}{m_{s}} \sum_{i=1}^{m_{s}} \chi_{[0, \delta)}\left(\rho\left(f_{1}^{i}\left(x_{\delta^{*}}\right), f_{1}^{i}\left(y_{\delta^{*}}\right)\right)\right)=0 .
$$

Similar to the proof of (i), one can find $c \in\{0,1, \cdots, m-1\}$ such that

$$
m_{s}^{c}=\{k m+c: k \in \mathbb{N}\} \cap\left\{m_{s}: s \in \mathbb{N}\right\}
$$

is an infinite set. Thus,

$$
\begin{aligned}
0 & =\lim _{8 \rightarrow \infty} \frac{1}{m_{s}^{c}} \sum_{i=1}^{m_{s}^{c}} \chi_{\left[0, \delta_{1}\right)}\left(\rho\left(f_{1}^{i}\left(x_{\delta^{*}}\right), f_{1}^{i}\left(y_{\delta^{*}}\right)\right)\right) \\
& =\lim _{8 \rightarrow \infty} \frac{1}{m_{s}^{c}+m-c} \sum_{i=1}^{m_{s}^{c}+m-c} \chi_{\left[0, \delta_{1}\right)}\left(\rho\left(f_{1}^{i}\left(x_{\delta^{*}}\right), f_{1}^{i}\left(y_{\delta^{*}}\right)\right)\right) \\
& =\lim _{8 \rightarrow \infty} \frac{1}{m_{s}^{c}+m-c} \sum_{i=1}^{m} \sum_{i=1}^{\frac{1}{m}\left(m_{s}^{c}+m-c\right)-1} \chi_{\left[0, \delta_{1}\right)}\left(\rho\left(f_{1}^{i m+j}\left(x_{\delta^{*}}\right), f_{1}^{i m+j}\left(y_{\delta^{*}}\right)\right)\right) \\
& \geq \sum_{j=1}^{m} \liminf _{s \rightarrow \infty} \frac{1}{m_{s}^{c}+m-c} \cdot \\
\frac{1}{m}\left(m_{s}^{c}+m-c\right)-1 & \chi_{\left[0, \delta_{1}\right)}\left(\rho\left(f_{1}^{i m+j}\left(x_{\delta^{*}}\right), f_{1}^{i m+j}\left(y_{\delta^{*}}\right)\right)\right)
\end{aligned}
$$

$$
\begin{aligned}
& \geq \liminf _{s \rightarrow \infty} \frac{1}{m} \cdot \frac{m}{m_{s}^{c}+m-c} \cdot \\
& \quad \sum_{i=0}^{\frac{1}{m}\left(m_{s}^{c}+m-c\right)-1} \chi_{\left[0, \delta_{1}\right)}\left(\rho\left(f_{1}^{(i+1) m}\left(x_{\delta^{*}}\right), f_{1}^{(i+1) m}\left(y_{\delta^{*}}\right)\right)\right) \\
& \geq \frac{1}{m} F_{\delta_{\delta^{*}} y_{\delta^{*}}}\left(\delta_{1}, f_{1, \infty}^{[m]}\right) \\
& \geq \frac{1}{m} F_{x_{\delta^{*} y_{\delta^{*}}}}\left(\delta^{*}, f_{1, \infty}^{[m]}\right)>0 .
\end{aligned}
$$

It is a contradiction.

So, $f_{1, \infty}^{[m]}$ is uniformly distributionally chaotic.

The proof is completed.

\section{Theorem 4.2}

If the mapping sequence $f_{1, \infty}$ is maximally distributionally chaotic, then for any $m \in \mathbb{N}, f_{1, \infty}^{[m]}$ is also maximally distributionally chaotic.

\section{Proof}

Let $S_{0}$ be a maximal distributionally scrambled set of $f_{1, \infty}$, the following will prove that $S_{0}$ is also a maximal distributionally scrambled set of $f_{1, \infty}^{[m]}, \forall m \in \mathbb{N}$.

(i) By the proof of Theorem 4.1, for any $x, y \in S_{0}: x \neq y$ and any $t>0$, one has $F_{x y}^{*}\left(t, f_{1, \infty}^{[m]}\right)=1$.

(ii) For any $x, y \in S_{0}: x \neq y$ and any $0<\delta<\operatorname{diamI}$, one can prove that

$$
F_{x y}\left(\delta, f_{1, \infty}^{[m]}\right)=\liminf _{k \rightarrow \infty} \frac{1}{k} \sum_{i=1}^{k} \chi_{[0, \delta)}\left(\rho\left(f_{1}^{[i m]}(x), f_{1}^{[i m]}(y)\right)\right)=0 .
$$

In fact, since $S_{0}$ is a maximal distributionally scrambled set of $f_{1, \infty}$, then $F_{x y}\left(\delta_{1}, f_{1, \infty}\right)=0$ for any $x, y \in S_{0}: x \neq y$ and any $\delta: 0<\delta<\operatorname{diamI}$.

Hypothetically, there exist $x_{\delta^{*}}, y_{\delta^{*}} \in S_{0}$ and $\delta^{*}=\frac{\delta}{2}$ such that $F_{x_{\delta^{*} y_{\delta^{*}}^{*}}}\left(\delta^{*}, f_{1, \infty}^{[m]}\right)>0$.

Since $F_{x_{\delta^{*}} \delta_{\delta^{*}}^{*}}\left(\delta, f_{1, \infty}\right)=0$, then there exists a strictly increasing sequence $\left\{m_{s}\right\}_{s \in \mathbb{N}}$ such that

$$
F_{x_{\delta^{*}} y_{\delta^{*}}^{*}}\left(\delta^{*}, f_{1, \infty}\right)=\lim _{s \rightarrow \infty} \frac{1}{m_{s}} \sum_{i=1}^{m_{s}} \chi_{[0, \delta)}\left(\rho\left(f_{1}^{i}\left(x_{\delta^{*}}\right), f_{1}^{i}\left(y_{\delta^{*}}\right)\right)\right)=0 .
$$

One can find $c \in\{0,1, \cdots, m-1\}$ such that

$$
m_{s}^{c}=\{k m+c: k \in \mathbb{N}\} \cap\left\{m_{s}: s \in \mathbb{N}\right\}
$$


is an infinite set. Then,

$$
\begin{aligned}
& 0=\lim _{s \rightarrow \infty} \frac{1}{m_{s}^{c}} \sum_{i=1}^{m_{s}^{c}} \chi_{[0, \delta)}\left(\rho\left(f_{1}^{i}\left(x_{\delta^{*}}\right), f_{1}^{i}\left(y_{\delta^{*}}\right)\right)\right) \\
& =\lim _{s \rightarrow \infty} \frac{1}{m_{s}^{c}+m-c} \sum_{i=1}^{m_{s}^{c}+m-c} \chi_{[0, \delta)}\left(\rho\left(f_{1}^{i}\left(x_{\delta^{*}}\right), f_{1}^{i}\left(y_{\delta^{*}}\right)\right)\right) \\
& =\lim _{s \rightarrow \infty} \frac{1}{m_{s}^{c}+m-c} \sum_{i=1}^{m} \sum_{i=0}^{\frac{1}{m}\left(m_{s}^{c}+m-c\right)-1} \chi_{[0, \delta)}\left(\rho\left(f_{1}^{i m+j}\left(x_{\delta^{*}}\right), f_{1}^{i m+j}\left(y_{\delta^{*}}\right)\right)\right) \\
& \geq \sum_{j=1}^{m} \liminf _{s \rightarrow \infty} \frac{1}{m_{s}^{c}+m-c} \sum_{i=0}^{\frac{1}{m}\left(m_{s}^{c}+m-c\right)-1} \chi_{[0, \delta)}\left(\rho\left(f_{1}^{i m+j}\left(x_{\delta^{*}}\right), f_{1}^{i m+j}\left(y_{\delta^{*}}\right)\right)\right) \\
& \geq \frac{1}{m} F_{x_{\delta^{*} y_{\delta^{*}}}}\left(\delta, f_{1, \infty}^{[m]}\right) \\
& \geq \frac{1}{m} F_{x_{\delta^{*} y_{\delta^{*}}}}\left(\delta^{*}, f_{1, \infty}^{[m]}\right)>0 .
\end{aligned}
$$

It is a contradiction.

So, $f_{1, \infty}^{[m]}$ is maximally distributionally chaotic.

The proof is completed.

The above two Theorems show that uniformly distributional chaos (or maximal distributional chaos) of $f_{1, \infty}$ is maintained under the compound case. While, if $\forall m \in \mathbb{N}, m \geq 2, f_{1, \infty}^{[m]}$ is uniformly distributional chaos (or maximal distributional chaos), whether $f_{1, \infty}$ is uniformly distributional chaos (or maximal distributional chaos) or not? To answer this question, three lemmas are given first.

Lemma 4.3 (Wu et al., 2013)

If the mapping sequence $\left(f_{n}\right)_{n=1}^{\infty}$ converges uniformly to $f$, then $\forall m \in \mathbb{N}, m \geq 2$, the mapping sequence $\left(f_{n}^{m}\right)_{n=1}^{\infty}$ converges uniformly to $f^{m}$.

Lemma 4.4 (Wu et al., 2013)

If the mapping sequence $\left(f_{n}\right)_{n=1}^{\infty}$ is equicontinuous, then $\forall m \in \mathbb{N}, m \geq 2$, the mapping sequence $\left(f_{n}^{m}\right)_{n=1}^{\infty}$ is equicontinuous.

Lemma 4.5(Wu et al., 2013)

If the mapping sequence $\left(f_{n}\right)_{n=1}^{\infty}$ is equicontinuous (or uniformly converges to $f$ ), then $\forall \varepsilon>0, \forall m \in \mathbb{N}$, there exist $\delta(\varepsilon)>0$ and $N(m) \in \mathbb{N}$ such that $\rho\left(f_{n}^{m}(x), f_{n}^{m}(y)\right)<\varepsilon \quad$ for $\quad \forall x, y \in I: \rho(x, y)<\delta(\varepsilon) \quad$ and $n \geq N(m)$.

Now the question posed above can be answered.

\section{Theorem 4.6}

If $\left(f_{n}\right)_{n=1}^{\infty}$ is equicontinuous (or uniformly converges to $f$ ) and $\forall m \in \mathbb{N}, m \geq 2, f_{1, \infty}^{[m]}$ is uniformly distributionally chaotic, then $f_{1, \infty}$ is uniformly distributionally chaotic.

\section{Proof.}

Let $D_{0}$ be a uniformly distributionally scrambled set of $f_{1, \infty}^{[m]}$.

If $F_{x, y}^{*}\left(\delta, f_{1, \infty}^{[m]}\right)=1$ for any $x, y \in D_{0}: x \neq y$ and any $\delta>0$, then, $F_{x, y}^{*}\left(t, f_{1, \infty}\right)=1$ for any $x, y \in D_{0}: x \neq y$ and any $t>0$.

In fact, for any $t>0$, by Lemma 4.5 , there exist $\delta: 0<\delta<t$ and $N \in \mathbb{N}$ such that, for any $x, y \in I: \rho(x, y)<\delta$ and any $k \geq N, \rho\left(f_{k}^{i}(x), f_{k}^{i}(y)\right)<t$ for any $i: 1 \leq i \leq m$. So, for any $j \geq N: \rho\left(f_{1}^{j m}(x), f_{1}^{j m}(y)\right)<\delta$ and any $i: 1 \leq i \leq m$, one has that

$$
\rho\left(f_{1}^{j m+i}(x), f_{1}^{j m+i}(y)\right)<t
$$

Then:

$$
\sum_{j=1}^{k} \chi_{[0, \delta)}\left(\rho\left(f_{1}^{j m}(x), f_{1}^{j m}(y)\right)\right)-N \leq \frac{1}{m} \sum_{j=1}^{(k+1) m} \chi_{[0, t)}\left(\rho\left(f_{1}^{i}(x), f_{1}^{j}(y)\right)\right) .
$$

Thus,

$$
\begin{aligned}
F_{x, y}^{*}\left(t, f_{1, \infty}\right) & =\limsup _{k \rightarrow \infty} \frac{1}{k} \sum_{j=1}^{k} \chi_{[0, t)}\left(\rho\left(f_{1}^{j}(x), f_{1}^{j}(y)\right)\right) \\
& =\limsup _{k \rightarrow \infty} \frac{1}{(k+1) m} \sum_{j=1}^{(k+1) m} \chi_{[0, t)}\left(\rho\left(f_{1}^{j}(x), f_{1}^{j}(y)\right)\right) \\
& =\limsup _{k \rightarrow \infty} \frac{1}{k m} \sum_{j=1}^{(k+1) m} \chi_{[0, t)}\left(\rho\left(f_{1}^{j}(x), f_{1}^{j}(y)\right)\right) \\
& =\limsup _{k \rightarrow \infty} \frac{1}{k}\left(\sum_{j=1}^{k} \chi_{[0, t)}\left(\rho\left(f_{1}^{j m}(x), f_{1}^{j m}(y)\right)\right)-N\right) \\
& =1 .
\end{aligned}
$$

If there exists a $s>0$ such that $F_{x, y}\left(s, f_{1, \infty}^{[m]}\right)=0$ for any $x, y \in D_{0}: x \neq y$, then there exists a $\delta>0$ such that $F_{x, y}\left(\delta, f_{1, \infty}\right)=0$ for any $x, y \in D_{0}: x \neq y$. 
In fact, for the above $s>0$, there exist $\delta$ : $0<\delta<s$ and $N_{1}$ $\in \mathbb{N}$ such that, for any $x, y \in I: \rho(x, y)<\delta$ and any $k \geq N_{1}$, $\rho\left(f_{k}^{i}(x), f_{k}^{i}(y)\right)<s$ for any $i: 1 \leq i \leq m$. So, for any $j \geq N_{1}: \rho\left(f_{1}^{j m}(x), f_{1}^{j m}(y)\right) \geq s$ and any $i: 1 \leq 1 \leq m$, one has that

$$
\rho\left(f_{1}^{j m-i}(x), f_{1}^{j m-i}(y)\right) \geq \delta .
$$

Then,

$$
\sum_{j=1}^{k} \chi_{[s, \infty)}\left(\rho\left(f_{1}^{j m}(x), f_{1}^{j m}(y)\right)\right)-N_{1} \leq \frac{1}{m} \sum_{j=1}^{k m} \chi_{[\delta, \infty)}\left(\rho\left(f_{1}^{j}(x), f_{1}^{j}(y)\right)\right) .
$$

And because

$$
\begin{aligned}
& \limsup _{k \rightarrow \infty} \frac{1}{k}\left(\sum_{j=1}^{k} \chi_{[s, \infty)}\left(\rho\left(f_{1}^{j m}(x), f_{1}^{j m}(y)\right)\right)\right) \\
& =1-\liminf _{k \rightarrow \infty} \frac{1}{k}\left(\sum_{j=1}^{k} \chi_{[0, s)}\left(\rho\left(f_{1}^{j m}(x), f_{1}^{j m}(y)\right)\right)\right) \\
& =1-F_{x, y}\left(s, f_{1, \infty}^{[m]}\right)=1 .
\end{aligned}
$$

Then,

$$
\underset{k \rightarrow \infty}{\limsup } \frac{1}{k}\left(\sum_{j=1}^{k} \chi_{[\delta, \infty)}\left(\rho\left(f_{1}^{j}(x), f_{1}^{j}(y)\right)\right)\right)=1 .
$$

Thus,

$$
F_{x, y}\left(\delta, f_{1, \infty}\right)=1-\limsup _{k \rightarrow \infty} \frac{1}{k}\left(\sum_{j=1}^{k} \chi_{[\delta, \infty)}\left(\rho\left(f_{1}^{j}(x), f_{1}^{j}(y)\right)\right)\right)=0 .
$$

So $f_{1, \infty}$ is uniformly distributional chaos.

\section{Theorem 4.7}

If $\left(f_{n}\right)_{n=1}^{\infty}$ is equicontinuous (or uniformly converges to $f$ ) an $\forall m \in \mathbb{N}, m \geq 2, f_{1, \infty}^{[m]}$ is maximally distributionally chaotic, then $f_{1, \infty}$ is maximally distributionally chaotic.

\section{Proof.}

Let $D_{0}$ be a maximal distributionally scrambled set of $f_{1, \infty}^{[m]}$. For any $x, y \in D_{0}: x \neq y$, one has

(i) If $F_{x, y}^{*}\left(\delta, f_{1, \infty}^{[m]}\right)=1$ for any $\delta>0$, then $F_{x, y}^{*}\left(t, f_{1, \infty}\right)=1$ for any $t>0$;

(ii) If $F_{x, y}\left(s, f_{1, \infty}^{[m]}\right)=0$ for any $0<s<$ diamI, then $F_{x, y}\left(\delta, f_{1, \infty}\right)=0$ for any $0<\delta<\operatorname{diamI}$.

The proof of upper distributional function for $f_{1, \infty}$ is the same as the proof of uniformly distributional chaos, so it is omitted. The following will lower upper distributional function for $f_{1, \infty}$.
In fact, for any $0<\delta<$ diamI, there exist s: $0<s<\delta$ and $N \in \mathbb{N}$ such that, for any $x, y \in I: \rho(x, y)$ and any $k \geq N$ $\rho\left(f_{k}^{i}(x), f_{k}^{i}(y)\right)<\delta$ for any $i: 1 \leq \mathrm{I} \leq m$, So, for any $j \geq N: \rho\left(f_{1}^{j m}(x), f_{1}^{j m}(y)\right)<s$ and any $i: 1 \leq \mathrm{I} \leq m$, one has that

$$
\rho\left(f_{1}^{j m+i}(x), f_{1}^{j m+i}(y)\right)<\delta .
$$

Then,

$$
\begin{aligned}
& \sum_{j=1}^{k} \chi_{[0, s)}\left(\rho\left(f_{1}^{j m}(x), f_{1}^{j m}(y)\right)\right)-N \\
\leq & \frac{1}{m} \sum_{j=1}^{(k+1) m} \chi_{[0, \delta)}\left(\rho\left(f_{1}^{j}(x), f_{1}^{j}(y)\right)\right)
\end{aligned}
$$

Thus,

$$
\begin{aligned}
F_{x, y}\left(\delta, f_{1, \infty}\right) & =\liminf _{k \rightarrow \infty} \frac{1}{k} \sum_{j=1}^{k} \chi_{[0, \delta)}\left(\rho\left(f_{1}^{j}(x), f_{1}^{j}(y)\right)\right) \\
& =\liminf _{k \rightarrow \infty} \frac{1}{(k+1) m} \sum_{j=1}^{(k+1) m} \chi_{[0, \delta)}\left(\rho\left(f_{1}^{j}(x), f_{1}^{j}(y)\right)\right) \\
& =\liminf _{k \rightarrow \infty} \frac{1}{k m} \sum_{j=1}^{(k+1) m} \chi_{[0, \delta)}\left(\rho\left(f_{1}^{j}(x), f_{1}^{j}(y)\right)\right) \\
& =\liminf _{k \rightarrow \infty} \frac{1}{k}\left(\sum_{j=1}^{k} \chi_{[0, \delta)}\left(\rho\left(f_{1}^{j m}(x), f_{1}^{j m}(y)\right)\right)-N\right)=0 .
\end{aligned}
$$

So $f_{1, \infty}$ is maximal distributional chaos.

The proof is completed.

In Theorem 4.6 and Theorem 4.7, the condition ' $\left(f_{n}\right)_{n=1}^{\infty}$ is equicontinuous (or uniformly converges to $f$ )' is indispensable. We give a counter example to show that, there is a compound system which is distributional chaos, but the original system is not. The following proof is for uniformly distributional chaos. Maximal distributional chaos is similar.

\section{Example 4.8}

Define metric $\rho: I \times I \rightarrow[0,1]$ as:

$$
\rho(x, y)\left\{\begin{array}{l}
0, x=y, \\
\frac{1}{2^{2 k}},[x]=[y] \equiv 0(\bmod 2), x \neq y \in\left(\sum_{j=1}^{2 k} b_{j}, \sum_{j=1}^{2 k+1} b_{j}\right), k \in \mathbb{N}, \\
1, \text { others. }
\end{array}\right.
$$

where, $b_{1}=2, b_{i}=2^{b_{1}+\ldots+b_{i-1}}$.

Let

$$
f_{1}=\frac{1}{2} x, f_{2}=2 x+1, \quad\left(f_{n}\right)_{n=1}^{\infty}=\left(f_{1}, f_{2}, f_{1}, f_{2}, \ldots\right),
$$

define functions $g_{i}=f_{2} \circ f_{1}(i \in \mathbb{N})$, then

(1) $g_{1, \infty}$ is not uniformly distributional chaos;

(2) $g_{1, \infty}^{[2]}$ is uniformly distributional chaos. 
Proof.

(1) $\forall x, y \in[0,+\infty)(x \neq y)$, the following prove that

$F_{x y}^{*}\left(\frac{1}{2}, g_{1, \infty}\right)=\limsup _{k \rightarrow \infty} \frac{1}{k} \sum_{i=1}^{k} \chi_{\left[0, \frac{1}{2}\right)}\left(\rho\left(g_{1}^{i}(x), g_{1}^{i}\right)(y)\right) \leq \frac{1}{2}$.

It can be proved in three cases.

(1a) if $[x] \neq[y]$, then $\forall i \in \mathbb{N}$,

$$
\left[g_{1}^{i}(x)\right]=[x+i] \neq[y+i]=\left[g_{1}^{i}(y)\right],
$$

so $\rho\left(g_{1}^{i}(x), g_{1}^{i}(y)\right)=1$. Therefore,

$$
\limsup _{k \rightarrow \infty} \frac{1}{k} \sum_{i=1}^{k} \chi_{\left[0, \frac{1}{2}\right)}\left(\rho\left(g_{1}^{i}(x), g_{1}^{i}(y)\right)\right)=0 .
$$

(1b) if $[x] \equiv[y] \equiv 0(\bmod 2)$, then $\forall i \in \mathbb{N}$, :

$\left[g_{1}^{2 i+1}(x)\right]=[x]+2 i+1=[y]+2 i+1=\left[g_{1}^{2 i+1}(y)\right] \equiv 1(\bmod 2)$,

so $\rho\left(g_{1}^{2 i+1}(x), g_{1}^{2 i+1}(y)\right)=1$.

Therefore,

$$
\limsup _{k \rightarrow \infty} \frac{1}{k} \sum_{i=1}^{k} \chi_{\left[0, \frac{1}{2}\right)}\left(\rho\left(g_{1}^{i}(x), g_{1}^{i}(y)\right)\right) \leq \limsup _{k \rightarrow \infty} \frac{\left[\frac{k}{2}\right]}{k}=\frac{1}{2} .
$$

(1c) if $[x] \equiv[y] \equiv 1(\bmod 2)$, at the same time, it was noted that $\left[g_{1}^{i}(x)\right]=\left[g_{1}^{i}(y)\right] \equiv 0(\bmod 2)$, by $(1 \mathrm{~b})$,

$$
\limsup _{k \rightarrow \infty} \frac{1}{k} \sum_{i=1}^{k} \chi_{\left[0, \frac{1}{2}\right)}\left(\rho\left(g_{1}^{i}(x), g_{1}^{i}(y)\right)\right) \leq \frac{1}{2} .
$$

Thus, $g_{1, \infty}$ is not uniformly distributional chaos.

(2) Denote $S=(0,1)$, one can proved that $S$ is a uniformly distributionally scrambled set of $g_{1, \infty}^{[2]}$.

(2a) For any $t>0$, take $n_{0} \in \mathbb{N}$, such that $\frac{1}{2^{2 n_{0}}}<t$. Let

$$
L_{n}=\sum_{j=1}^{n} b_{j} \cdot \forall x, y \in S(x \neq y), \forall n \geq n_{0}, \forall_{i} \in\left[\frac{L_{2 n}}{2}, \frac{L_{2 n+1}}{2}-1\right),
$$

since

$$
\begin{aligned}
& \left(g_{1, \infty}^{[2]}\right)_{1}^{i}(x)=x+2 i \in\left[L_{2 n}, L_{2 n+1}\right), \\
& \left(g_{1, \infty}^{[2]}\right)_{1}^{i}(y)=y+2 i \in\left[L_{2 n}, L_{2 n+1}\right),
\end{aligned}
$$

and

$$
\left[\left(g_{1, \infty}^{[2]}\right)_{1}^{i}(x)\right]=\left[\left(g_{1, \infty}^{[2]}\right)_{1}^{i}(y)\right]=2 i,
$$

then,

$$
\rho\left(\left(g_{1, \infty}^{[2]}\right)_{1}^{i}(x),\left(g_{1, \infty}^{[2]}\right)_{1}^{i}(y)\right)=\rho(x+2 i, y+2 i)=\frac{1}{2^{2 n}} \leq \frac{1}{2^{n_{0}}}<t .
$$

Therefore,

$$
\frac{1}{\frac{L_{2 n+1}}{2}} \sum_{i=1}^{\frac{L_{2 n+1}}{2}} \chi_{[0, t)}\left(\rho\left(\left(g_{1, \infty}^{[2]}\right)_{1}^{i}(x),\left(g_{1, \infty}^{[2]}\right)_{1}^{i}(y)\right)\right) \geq \frac{\frac{L_{2 n+1}-L_{2 n}}{2}-1}{\frac{L_{2 n+1}}{2}} .
$$

So,

$$
\begin{aligned}
& 1 \geq F_{x, y}^{*}\left(t, g_{1, \infty}^{[2]}\right) \\
& =\limsup _{k \rightarrow \infty} \frac{1}{k} \sum_{i=1}^{k} \chi_{[0, t)}\left(\rho\left(\left(g_{1, \infty}^{[2]}\right)_{1}^{i}(x),\left(g_{1, \infty}^{[2]}\right)_{1}^{i}(y)\right)\right) \\
& \geq \limsup _{n \rightarrow \infty} \frac{1}{\frac{L_{2 n+1}}{2}} \sum_{i=1}^{\frac{L_{2 n+1}}{2}} \chi_{[0, t)}\left(\rho\left(\left(g_{1, \infty}^{[2]}\right)_{1}^{i}(x),\left(g_{1, \infty}^{[2]}\right)_{1}^{i}(y)\right)\right) \\
& \geq \limsup _{n \rightarrow \infty} \frac{\frac{L_{2 n+1}-L_{2 n}}{2}-1}{\frac{L_{2 n+1}}{2}} \\
& =\limsup _{n \rightarrow \infty} \frac{\frac{2^{b_{1}+b_{2}+\ldots+b_{2 n}}}{2}-1}{\frac{b_{1}+b_{2}+\ldots+b_{2 n}+2^{b_{1}+b_{2}+\ldots+b_{2 n}}}{2}} \\
& =1 \text {. }
\end{aligned}
$$

(2b) Put

$$
t_{0}=\frac{1}{2}, \forall x, y \in S(x \neq y), \forall n \in \mathbb{N}, \forall i \in\left[\frac{L_{2 n+1}}{2}, \frac{L_{2 n+2}}{2}-1\right),
$$

Since

$$
\left(g_{1, \infty}^{[2]}\right)_{1}^{i}(x) \neq\left(g_{1, \infty}^{[2]}\right)_{1}^{i}(y) \in\left[L_{2 n+1}, L_{2 n+2}\right),
$$

then 


$$
\rho\left(\left(g_{1, \infty}^{[2]}\right)_{1}^{i}(x),\left(g_{1, \infty}^{[2]}\right)_{1}^{i}(y)\right)=1 .
$$

So,

$$
\frac{1}{\frac{L_{2 n+2}}{2}} \sum_{i=1}^{\frac{L_{2 n+2}}{2}} \chi_{\left[0, \frac{1}{2}\right.}\left(\rho\left(\left(g_{1, \infty}^{[2]}\right)_{1}^{i}(x),\left(g_{1, \infty}^{[2]}\right)_{1}^{i}(y)\right)\right) \leq \frac{\frac{L_{2 n+2}}{2}-\left(\frac{b_{2 n+2}}{2}-1\right)}{\frac{L_{2 n+2}}{2}} .
$$

One can obtain that,

$$
\begin{aligned}
& 0 \leq F_{x, y}\left(\frac{1}{2}, g_{1, \infty}^{[2]}\right) \\
& =\liminf _{k \rightarrow \infty} \frac{1}{k} \sum_{i=1}^{k} \chi_{\left[0, \frac{1}{2}\right)}\left(\rho\left(\left(g_{1, \infty}^{[2]}\right)_{1}^{i}(x),\left(g_{1, \infty}^{[2]}\right)_{1}^{i}(y)\right)\right) \\
& \leq \liminf _{n \rightarrow \infty} \frac{1}{\frac{L_{2 n+2}}{2}} \sum_{i=1}^{\frac{L_{2 n+2}}{2}} \chi_{\left[0, \frac{1}{2}\right)}\left(\rho\left(\left(g_{1, \infty}^{[2]}\right)_{1}^{i}(x),\left(g_{1, \infty}^{[2]}\right)_{1}^{i}(y)\right)\right) \\
& \leq \liminf _{n \rightarrow \infty} \frac{\frac{L_{2 n+2}}{2}-\left(\frac{b_{2 n+2}}{2}-1\right)}{\frac{L_{2 n+2}}{2}} \\
& =\liminf _{n \rightarrow \infty} \frac{\frac{b_{1}+b_{2}+\ldots+b_{2 n+1}}{2}+1}{\frac{b_{1}+b_{2}+\ldots+b_{2 n+1}+2^{b_{1}+b_{2}+\ldots b_{2 n+1}}}{2}}=0 .
\end{aligned}
$$

Thus, $g_{1, \infty}^{[2]}$ is uniformly distributional chaos.

\section{Acknowledgment}

This work was funded by the Opening Project of Key Laboratory of Higher Education of Sichuan Province for Enterprise Informationalization and Internet of Things (No. 2020WZJ01), the Scientific Research Project of Sichuan University of Science and Engineering (No. 2020RC24), and the Graduate student Innovation Fund (Nos. y2021100, cx2020188).

\section{Ethics}

The study was approved by Sichuan University of Science and Engineering and Key Laboratory of Higher Education of Sichuan Province for Enterprise Informationalization and Internet of Things. All authors agree to publish. There is no conflict of interest.

\section{References}

Akin, E., \& Kolyada, S. (2003). Li-Yorke sensitivity. Nonlinearity, 16(4), 1421. https://iopscience.iop.org/article/10.1088/0951$7715 / 16 / 4 / 313 /$ meta
Balibrea, F., Smital, J., \& Štefánková, M. (2005). The three versions of distributional chaos. Chaos, Solitons \& Fractals, 23(5), 1581-1583. doi.org/10.1016/j.chaos.2004.06.011

Banks, J., Brooks J., Cairns G., Davis G., \& Stacey P. (1992). On Devaney's definition of chaos, American Mathematical Monthly, 99(4): 332-334. doi.org/10.2307/2324899.

Dvorakova, J. (2011). On a problem of iteration invariants for distributional chaos. Communications in Nonlinear Science and Numerical Simulation, 17(2): 785-787. doi.org/10.1016/j.cnsns.2011.06.015

Kolyada, S., \& Trofimchuk, S. (2004). On minimality of nonautonomous dynamical systems. Nonlinear Oscillations, 7(1), 83-89.

https://link.springer.com/article/10.1023/B:NONO.0 000041798.79176 .94

Kolyada, S., Misiurewicz, M., \& Snoha, L. U. (1999). Topological entropy of nonautonomous piecewise monotone dynamical systems on the interval. Fundamenta Mathematicae, 160, 161-181. 10.4064/fm-160-2-161-181.

Li, T. Y., \& Yorke, J. (1975). Period three implies chaos. Amer Math Monthly, 1975, 82(10), 985-992.

Liao, G., Chu, Z., \& Fan, Q. (2009). Relations between mixing and distributional chaos. Chaos, Solitons \& Fractals, 41(4), 1994-2000. doi.org/10.1016/j.chaos.2008.08.003

Wu, X. X., \& Zhu, P. Y., (2013). Chaos in a class of non-autonomous discrete systems. Applied Mathematics Letters, 26(04): 431-436. doi.org/10.1016/j.aml.2012.11.003

Martínez-Giménez, F., Oprocha, P., \& Peris, A. (2009). Distributional chaos for backward shifts. Journal of Mathematical Analysis and Applications, 351(2), 607-615. doi.org/10.1016/j.jmaa.2008.10.049

Oprocha, P. (2009). Distributional chaos revisited. Transactions of the American Mathematical Society, 361(9), 4901-4925.

https://www.ams.org/journals/tran/2009-36109/S0002-9947-09-04810-7/

Li, R. (2011). A note on the three versions of distributional chaos. Communications in Nonlinear Science and Numerical Simulation, 16(4), 1993-1997. doi.org/10.1016/j.cnsns.2010.08.014

Ruette, S. (2005). Dense chaos for continuous interval maps. Nonlinearity, 18(4), 1691. https://iopscience.iop.org/article/10.1088/09517715/18/4/015/meta

Schweizer, B., \& Smital, J. (1994). Measures of chaos and a spectral decomposition of dynamical systems on the interval. Transactions of the American Mathematical Society, 344(2), 737-754.

https://www.ams.org/journals/tran/1994-34402/S0002-9947-1994-1227094-X/ 
Shao, H., Shi, Y., \& Zhu, H. (2018). On distributional chaos in non-autonomous discrete systems. Chaos, Solitons \& Fractals, 107, 234-243. doi.org/10.1016/j.chaos.2018.01.005

Smítal, J., \& Štefánková, M. (2004). Distributional chaos for triangular maps. Chaos, Solitons \& Fractals, 21(5), 1125-1128. doi.org/10.1016/j.chaos.2003.12.105

Snoha, L. (1990). Generic chaos. Commentationes Mathematicae Universitatis Carolinae, 31(4), 793-810. https://dml.cz/bitstream/handle/10338.dmlcz/106915 /CommentatMathUnivCarol_031-1990-4_20.pdf
Snoha, L. U. (1992). Dense chaos. Comment. Math. Univ. Carolin, 33(4), 747-752.

https://dml.cz/handle/10338.dmlcz/118547

Wu, X., \& Chen, G. (2013). On the invariance of maximal distributional chaos under an annihilation operator. Applied Mathematics Letters, 26(12), 1134-1140. doi.org/10.1016/j.aml.2013.06.011

Wu, X., Zhu, P., \& Lu, T. (2013). Uniform distributional chaos for weighted shift operators. Applied Mathematics Letters, 26(1), 130-133.

doi.org/10.1016/j.aml.2012.04.008 